\title{
Lipoprotein Metabolism during Acute Inhibition of Lipoprotein Lipase in the Cynomolgus Monkey
}

Ira J. Goldberg, Ngoc-Anh Le, Henry N. Ginsberg, Ronald M. Krauss, and Frank T. Lindgren

Department of Medicine, Columbia University College of Physicians and Surgeons, New York, New York 10032; Division of Arteriosclerosis, Mount Sinai School of Medicine, New York, New York 10029; and Lawrence Berkeley Laboratory,

Donner Laboratory, University of California, Berkeley, California 94720

\section{Abstract}

To clarify the role of lipoprotein lipase (LPL) in the catabolism of nascent and circulating very low density lipoproteins (VLDL) and in the conversion of VLDL to low density lipoproteins (LDL), studies were performed in which LPL activity was inhibited in the cynomolgus monkey by intravenous infusion of inhibitory polyclonal or monoclonal antibodies. Inhibition of LPL activity resulted in a three- to fivefold increase in plasma triglyceride levels within 3 h. Analytical ultracentrifugation and gradient gel electrophoresis demonstrated an increase predominantly in more buoyant, larger VLDL $\left(\mathbf{S}_{\mathbf{f}}\right.$ 400-60). LDL and high density lipoprotein (HDL) cholesterol levels fell during this same time period, whereas triglyceride in LDL and HDL increased. Kinetic studies, utilizing radiolabeled human VLDL, demonstrated that LPL inhibition resulted in a marked decrease in the catabolism of large $\left(S_{f}\right.$ 400-100) VLDL apolipoprotein $B$ (apoB). The catabolism of more dense VLDL $\left(S_{\mathrm{f}}\right.$ 60-20) was also inhibited, although to a lesser extent. However, there was a complete block in the conversion of tracer in both $S_{f}$ 400-100 and 60-20 VLDL apoB into LDL during LPL inhibition. Similarly, endogenous labeling of VLDL using $\left[{ }^{3} \mathrm{H}\right]$ leucine demonstrated that in the absence of LPL, no radiolabeled apoB appeared in LDL. We conclude that although catabolism of dense VLDL continues in the absence of LPL, this enzyme is required for the generation of LDL.

\section{Introduction}

Type I hyperlipoproteinemia is a genetic disorder characterized by the lack of lipoprotein lipase (LPL) ${ }^{1}$ activity in tissues and postheparin plasma (PHP) and the presence of fasting hyperchylomicronemia (1). In 1960, Havel and Gordon demonstrated that LPL activity was deficient in these patients by showing that their PHP lacked the enzymatic activity needed to hydrolyze triglyceride (TG) in chylomicrons (2). Although

Address correspondence to Ira J. Goldberg, M.D., Department of Medicine, Columbia University College of Physicians and Surgeons, 630 West 169th St., New York, NY 10032.

Received for publication 27 May 1987 and in revised form 8 September 1987.

1. Abbreviations used in this paper: apoB, apolipoprotein B; FFA, free fatty acid; HTGL, hepatic triglyceride lipase; LPL, lipoprotein lipase; PHP, postheparin plasma; TG, triglyceride; VLDL, very low density lipoprotein.

J. Clin. Invest.

(c) The American Society for Clinical Investigation, Inc.

$0021-9738 / 88 / 02 / 0561 / 08 \$ 2.00$

Volume 81, February 1988, 561-568 investigations of this human disorder and subsequent animal studies (3) have conclusively shown that chylomicron metabolism requires initial hydrolysis by LPL to produce remnant particles which are then removed from the circulation by the liver, the role(s) of LPL in metabolism of very low density lipoproteins (VLDL) is less certain. Studies of subjects with type I hyperlipoproteinemia employing endogenous labeling of TG using radioactive glycerol or fatty acids have demonstrated that, in the absence of LPL, VLDL TG is removed from the circulation at rates comparable to that of some subjects with normal amounts of this enzyme $(4,5)$. Subsequent studies by Nicoll and Lewis confirmed that VLDL were catabolized in LPL deficient subjects (6). By contrast, the finding that subjects with type IV hyperlipoproteinemia could develop fasting chylomicronemia (type $\mathrm{V}$ hyperlipoproteinemia) when their primary disorder was exacerbated suggested that both VLDL and chylomicrons share a common, saturable catabolic pathway (7). It has been hypothesized that whereas VLDL and chylomicrons may both interact first with LPL during normal metabolism, chylomicron metabolism may be restricted to the LPL initiated pathway, whereas VLDL have other routes of catabolism available to them. Previous work by us (8) and others $(9,10)$ suggested that one alternative pathway for VLDL catabolism involves hepatic triglyceride lipase (HTGL).

Epidemiologic studies have shown a positive correlation between circulating levels of low density lipoproteins (LDL) and the development of coronary atherosclerosis in humans. LDL are produced in the circulation by stepwise hydrolysis of triglyceride in the core of VLDL and loss of surface lipid and proteins resulting in the creation of denser, cholesterol-rich lipoproteins. Both in vitro and in vivo studies have demonstrated that LPL is important, but perhaps not essential, for the production of LDL. Deckelbaum et al. have produced LDLlike particles by incubating VLDL with purified bovine milk LPL (11). Humans with a deficiency of LPL have low circulating levels of LDL (1), which is thought to result from their inability to hydrolyze the triglyceride in precursor VLDL. Several lines of evidence suggest that alternative routes of production of LDL, which do not require LPL, are operative in normals and in some patients with disorders of lipoprotein metabolism. HTGL has been shown to hydrolyze VLDL triglyceride $(8,12)$ and thus this enzyme may be involved in an alternative pathway for conversion of VLDL to LDL. Kinetic studies of lipoprotein metabolism in humans and nonhuman primates have suggested that in some circumstances LDL may be independently secreted from the liver (13-15). Thus, LDL production appears to be heterogenous and may not always require the action of LPL.

A number of laboratories have used nonhuman primates for studies of experimental atherosclerosis and the effects of diet on circulating lipoproteins. In previous studies, we have 
shown that the cynomolgus monkey catabolizes both homologous and human VLDL at similar rates, and, in a manner analogous to the metabolic pathways observed in humans, converts VLDL into LDL (15). Furthermore, the monkey possesses a number of other similarities to humans including the presence of both postheparin lipases (16) and cholesteryl ester transfer protein activity $(17,18)$. In studies analogous to those which we performed previously to study the physiological roles of HTGL (8), we now report investigations carried out in the cynomolgus monkey to define the role of LPL in VLDL catabolism and in the production of LDL.

\section{Methods}

Animals. The protocols and procedures for these studies were reviewed and approved by the Columbia University Health Sciences Division Institutional Animal Care and Use Committee. Adult female cynomolgus monkeys weighing 3-4 $\mathrm{kg}$ were maintained on a chow diet containing 5\% fat (Ralston Purina, St. Louis, MO). The animals were fasted for 12-14 h before each study. Monkeys were anesthetized with intramuscular ketamine $(10 \mathrm{mg} / \mathrm{kg})$, removed from their cages, and maintained under ketamine anesthesia for the duration of the study. Additional ketamine was given intramuscularly $(5 \mathrm{mg} / \mathrm{kg})$ as needed every 30-60 min. All blood samples were obtained from the femoral veins by percutaneous venipuncture using a 25 -gauge needle attached to a syringe coated with a solution of EDTA to prevent the blood from clotting. A 20-gauge plastic intravenous catheter was inserted into the popliteal vein of each monkey for infusion of the antibody. In those studies in which radioactive tracers were injected, an additional catheter, a 21-gauge scalp vein needle, was inserted into either the opposite popliteal vein or into an axillary vein. After injection of the tracers, the scalp vein needle was flushed with $5 \mathrm{ml}$ of sterile saline and then removed. The animals did not appear distressed during the studies or during the evening after they were returned to their cages.

Due to limitations in the amount of blood which could be taken from each animal, separate monkeys were used for the studies of lipoprotein mass and for the determination of apolipoprotein B (apoB) kinetics. No animal received more than one study requiring injections of antibody. Some animals underwent two kinetic studies using the same VLDL preparations. In these instances, the initial study was performed without antibody infusion, and the second study was done during antibody infusion $3 \mathrm{~d}$ later. We have previously reported the effects of infusion of nonimmune globulins on lipoprotein levels and VLDL catabolism in this species (8). Control animals receiving no antibody were studied to verify that the changes in lipids were not due to the effects of the anesthesia.

A total of 12 monkeys were used for these studies. Their plasma cholesterol and TG levels were measured by enzymatic methods using the ABA 100 Autoanalyzer (Abbott Laboratories, Chicago, IL), and HDL cholesterol was measured after precipitation of apoB containing lipoproteins by addition or magnesium-dextran sulfate to plasma (19) (Table I).

Antibody preparation. We have previously reported the production of specific anti-human milk LPL polyclonal and monoclonal antibodies which inhibit the hydrolytic activity of the enzyme $(20,21)$. The polyclonal goat anti-LPL IgG preparation used in these studies was prepared in a manner similar to that previously reported for antiHTGL IgG (8). The IgG was precipitated by sodium sulfate and eluted through a column containing monkey plasma bound to Sepharose (Pharmacia Fine Chemicals, Piscataway, NJ) to remove antibodies not directed against proteins found primarily in PHP. Monoclonal antiLPL IgG was prepared from ascites collected from mice that had received two intraperitoneal injections of 2, 6, 10, 14-tetramethylpentadecane (pristane) followed by an injection of 5 million hybridoma cells. The IgG was isolated by precipitation with $\left(\mathrm{NH}_{4}\right)_{2} \mathrm{SO}_{4}$ followed by chromatography on diethyleaminoethyl Sepharose (Pharmacia
Table I. Plasma Lipid Levels of Monkeys Studied

\begin{tabular}{llll}
\hline Monkey & Cholesterol & TG & HDL cholesterol \\
\hline & $m g / d l$ & $m g / d l$ & $m g / d l$ \\
1 & 92 & 63 & 34 \\
2 & 95 & 76 & 30 \\
3 & 144 & 44 & 27 \\
4 & 125 & 75 & 15 \\
5 & 125 & 41 & 37 \\
6 & 170 & 70 & 34 \\
7 & 149 & 27 & 35 \\
8 & 111 & 51 & 35 \\
9 & 121 & 31 & 27 \\
10 & 116 & 67 & ND \\
11 & 166 & 67 & 63 \\
12 & 136 & 92 & 49 \\
\end{tabular}

Fine Chemicals) (22). The resulting monoclonal IgG preparations contained $0.5-2 \mathrm{mg} / \mathrm{ml}$ of protein.

Both the polyclonal and monoclonal antibodies were shown to inhibit cynomolgus monkey PHP LPL activity and not to inhibit HTGL activity. After an overnight fast, monkey PHP was obtained 10 min after intravenous injection of $100 \mathrm{U} / \mathrm{kg}$ body wt of heparin (Invenex, LyphoMed, Inc., Rosemont, IL). HTGL activity was measured using $200 \mu \mathrm{l}$ of a gum arabic stabilized TG emulsion in $1.25 \mathrm{M} \mathrm{NaCl}$ as described previously (16). LPL activity was measured using the emulsion described by Nilsson-Ehle and Schotz (23), and HTGL was inhibited using a specific antiserum (8). Monkey PHP was mixed with an equal volume of buffer ( $10 \mathrm{mM}$ sodium phosphate, $150 \mathrm{mM} \mathrm{NaCl}, \mathrm{pH}$ 7.4) containing dilutions of monoclonal anti-LPL IgG or anti-LPL antiserum, allowed to sit on ice for $5 \mathrm{~min}$, and then added to the emulsion. Enzyme activity was determined as the amount of free fatty acids (FFA) liberated during the 1-h incubation. The amount of activity remaining in the antibody-treated samples was compared with that in the sample containing no antibody.

Antibody infusions. For studies of lipoprotein mass, the animals received intravenous injections of $200 \mathrm{mg}$ of polyclonal or $4-8 \mathrm{mg}$ of monoclonal IgG at the initiation of the study and additional injections containing an equal mass of IgG every hour. The amounts of IgG were chosen to contain sufficient specific IgG to inhibit more than twice the amount of LPL which would have been expected in monkey plasma after intravenous heparin administration. At the completion of the study $(3 \mathrm{~h})$, a sample of monkey plasma was collected and tested to demonstrate that inhibitory levels of anti-LPL IgG were present in the circulation. Because these animals were receiving antibody every hour, this final sample at the conclusion of the study was obtained $60 \mathrm{~min}$ after the last antibody injection, at a time when the circulating levels of antibody should have been at the lowest level. In the studies of lipoprotein kinetics, after an initial blood sample, the monkeys received 4 $\mathrm{mg}$ of monoclonal $\mathrm{IgG}$ followed by $2 \mathrm{mg}$ of the monoclonal $\mathrm{IgG}$ every 30 min for the duration of the 6-h study.

Lipoprotein mass. TG and cholesterol were measured in plasmas obtained during control and experimental studies. For studies of lipoprotein profiles a $1-\mathrm{ml}$ sample was obtained before antibody infusion, and 6-ml blood samples were obtained at baseline, 1 and $3 \mathrm{~h}$. In some studies of lipoprotein kinetics, aliquots of hourly samples were also measured for TG and cholesterol. VLDL $(n=3)$, LDL $(n=3)$, and HDL $(n=6)$ cholesterol and TG were measured on lipoproteins isolated by ultracentrifugation before and after LPL inhibition.

Analytical ultracentrifugation of the plasma from three experimental animals was performed at the Donner Laboratory, University of California, Berkeley. The concentrations of the serum lipoproteins and their distribution as a function of flotation rate $\left(S_{f}\right.$ or $\left.F_{1.20}\right)$ were deter- 
mined by computerized analysis of data from the Schlieren patterns of analytical ultracentrifuge runs at $52,640 \mathrm{rpm}$ of the isolated fraction containing VLDL, IDL, and LDL in a baseline solution of $d 1.063$ $\mathrm{g} / \mathrm{ml}$ and the isolated HDL in a solution of $d 1.20 \mathrm{~g} / \mathrm{ml}(24)$. The flotation rates determined by a " $\mathrm{F} \mu$ vs. $\mathrm{p}$ " technique are expressed in Svedbergs $\left(10^{-15} \mathrm{~cm} \mathrm{~S}^{-1} \mathrm{dyn}^{-1} \mathrm{~g}^{-1}\right)$ and are corrected for concentration dependence and to standard conditions (temperature, $26^{\circ} \mathrm{C}$ ). The $d$ $<1.063 \mathrm{~g} / \mathrm{ml}$ plasma fractions from two of these animals and from one animal injected with control IgG were also used for gradient gel electrophoresis using a gradient of $2-16 \%$ acrylamide as described previously $(25,26)$. The gels were stained with oil red $O$ and the distribution of lipoproteins was quantitated by scanning of the gel using a Transidyne RFT densitometer. Samples obtained during studies of HTGL inhibition (8) and analyzed by this technique were compared with those obtained during LPL inhibition.

Studies of VLDL apoB kinetics. $100 \mathrm{ml}$ of plasma obtained from a normal human volunteer was used to isolate VLDL by ultracentrifugation at $d 1.006$ in an SW 28 rotor at $26,000 \mathrm{rpm}, 48 \mathrm{~h}, 10^{\circ} \mathrm{C}$ in a L5-75 ultracentrifuge (Beckman Instruments, Palo Alto, CA). The VLDL was concentrated by a second ultracentrifugation in an SW 60 rotor at $55,000 \mathrm{rpm}$ for $18 \mathrm{~h}$. After dialysis against $0.15 \mathrm{M} \mathrm{NaCl}, \mathrm{pH}$ 7.4 the VLDL was radioiodinated with ${ }^{125}$ I (Amersham Corp., Arlington Heights, IL) by the method of MacFarlane (27) as modified by Bilheimer et al. (28). The radioiodinated lipoproteins were extensively dialyzed against $0.15 \mathrm{M} \mathrm{NaCl}, \mathrm{pH} 7.4,0.01 \%$ EDTA to remove free iodine. The iodinated VLDL preparations were used within $4 \mathrm{~d}$ of labeling. The injected VLDL contained $10-20 \mu \mathrm{Ci}$ of radioactivity. Animals received a few drops of a saturated solution of potassium iodide in their drinking water for the week before each turnover study to prevent thyroidal uptake of radioactive iodine.

For studies of the kinetics of subpopulations of VLDL, blood ( 300 $\mathrm{ml}$ ) was obtained after an overnight fast from healthy normolipidemic human volunteers. The plasma was obtained, and the VLDL was isolated at $d 1.006$ in a SW 28 rotor spun at $26,000 \mathrm{rpm}, 48 \mathrm{~h}, 10^{\circ} \mathrm{C}$. Subpopulations of VLDL were isolated by centrifugation through a discontinuous gradient according to the method of Lindgren et al. (24) and were then concentrated by centrifugation in a $50.3 \mathrm{Ti}$ rotor containing $2-\mathrm{ml}$ inserts at $40,000 \mathrm{rpm}$ for $18 \mathrm{~h}$. Buoyant VLDL $\left(\mathrm{S}_{\mathrm{f}}\right.$ 400-100) was radioiodinated with ${ }^{125} I$ and dense $\left(S_{\mathrm{f}} 60-20\right)$ VLDL was radioiodinated with ${ }^{131} \mathrm{I}$.

After intravenous injection of radioiodinated VLDL, blood samples $(2.0-2.5 \mathrm{ml})$ were obtained at either 2 or $5 \mathrm{~min}$ and at 15 and 30 min and $1,2,3,4$, and $6 \mathrm{~h}$. All blood samples were collected into syringes containing EDTA and stored at $4^{\circ} \mathrm{C}$ after centrifugation to remove cells. A 100- $\mu$ l aliquot of plasma was set aside for cholesterol and TG measurements and $1 \mathrm{ml}$ of plasma was used to isolate VLDL $(d<1.006)$, IDL (1.006-1.019), LDL (1.019-1.063), and (in some studies) HDL (1.063-1.21) by ultracentrifugation in the $50.3 \mathrm{Ti}$ rotor at $39,000 \mathrm{rpm}, 24 \mathrm{~h}, 10^{\circ} \mathrm{C} \mathrm{(29).}$

ApoB radioactivity and specific activity in VLDL, IDL, and LDL was determined using the method of Le et al. (30). Samples $(0.9 \mathrm{ml})$ were delipidated sequentially with acetone $(3 \mathrm{ml})$ and isopropyl alcohol $(3 \mathrm{ml})$. The protein was solubilized with $1 \mathrm{ml}$ of $9 \mathrm{M}$ tetramethylurea (TMU) (Burdick-Jackson Laboratories, Muskegon, MI) at room temperature for $1 \mathrm{~h}$. ApoB was selectively precipitated by addition of 1 $\mathrm{ml}$ of water and centrifugation at $300 \mathrm{~g}$ for $45 \mathrm{~min}$. The supernatant was aspirated and the pellet was washed with $3 \mathrm{ml}$ of water to remove any remaining TMU. Radioactivity was determined using an autogamma scintillation spectrometer (Packard Instruments, Inc., Downing Grove, IL) and, when necessary, corrections were made for crossover of radioactivity from ${ }^{131} I$ as measured in the ${ }^{125} I$ channel. Protein in each tube was determined (31) and the specific radioactivity of apoB was calculated. In all studies total apoB radioactivity per milliliter of plasma in VLDL, IDL, and LDL was calculated and the amount of apoB radioactivity was compared with that in the initial VLDL sample ( 2 or $5 \mathrm{~min}$ ). Reisolation of the tracer demonstrated that $>90 \%$ of the label was recoverable as VLDL with $2-2.2 \%$ and $3.2-4.4 \%$ of the radioactivity recovered in IDL and LDL, respectively.
Studies of endogenously labeled $A p o B$. We have previously used $\left[{ }^{3} \mathrm{H}\right]$ leucine to endogenously label apoB in the cynomolgus monkey and have compared the catabolism of $\left[{ }^{3} \mathrm{H}\right]$ leucine and ${ }^{131} \mathrm{I}$-labeled VLDL (32). In the present studies, monkeys received an intravenous injection of $1 \mathrm{mCi}$ of $\left[{ }^{3} \mathrm{H}\right]$ leucine $15 \mathrm{~min}$ after the initial infusion of anti-LPL monoclonal IgG. Blood sampling, repeated boluses of IgG, lipoprotein and apoB isolation were performed as described above.

\section{Results}

The titration curves of the antiserum and monoclonal IgG against monkey LPL and HTGL are shown in Fig. 1. 50\% inhibition of LPL activity was achieved using $\sim 20 \mathrm{ng}$ of IgG or a dilution of $>1$ to 100 of antiserum. This inhibition is similar to that achieved when these antibodies were used to inhibit human PHP LPL $(20,21)$. Neither antibody inhibited HTGL activity.

In three animals whose plasma was used for studies of the effect of LPL inhibition on lipoprotein mass (No. 1, 4, and 6) and in which no radioactive tracers were injected, monkey plasma obtained at the completion of the study was assessed for the presence of circulating antibodies to LPL. The addition of $10 \mu \mathrm{l}$ of monkey plasma to $10 \mu \mathrm{l}$ of human postheparin plasma resulted in a 73,86 , and $73 \%$ reduction in LPL enzymatic activity for the three monkeys, respectively. Control monkey plasma did not inhibit LPL activity. Thus, injection of anti-LPL antibodies as described in Methods produced and maintained high circulating levels of anti-LPL antibody for the duration of the studies. These circulating antibodies should have interacted with endothelial cell-associated LPL, which was exposed to the plasma and should have inhibited the hydrolytic activity of the enzyme.

Changes in lipoprotein mass. Infusion of anti-LPL antibodies resulted in a dramatic increase in the levels of plasma TG in the monkey (Fig. 2) with no change or a small decrease in total plasma cholesterol. After $3 \mathrm{~h}$ of LPL inhibition a threeto fivefold increase in TG concentration had occurred. This increase was predominantly due to an increase in VLDL TG levels (Table II). Whereas VLDL cholesterol levels also increased, the ratio of TG to cholesterol in this lipoprotein increased, suggesting that there was an increase in larger, TGrich VLDL. This was confirmed by both analytical ultracentrifugation and gradient gel electrophoresis. As shown in Fig. 3 and Table III, the increase in VLDL was confined to more buoyant lipoproteins, $S_{\mathrm{f}}>60$. These particles, as expected, were larger on gradient gel electrophoresis. As depicted in Fig.

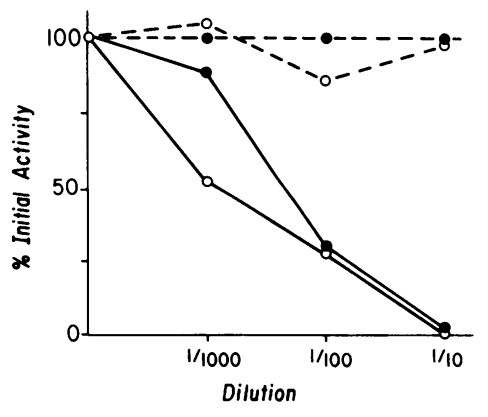

Figure 1. Titration of polyclonal (•) and monoclonal (o) antibodies against monkey LPL (solid line) and HTGL (dashed line). Cynomolgus monkey PHP containing LPL (8.8 $\mu \mathrm{mol}$ FFA/ml per $\mathrm{h}$ ) and HTGL (4.4 activities $\mu$ mol/FFA/ $\mathrm{ml}$ per $\mathrm{h}$ ) activities was mixed with an equal volume of phosphate buffered saline containing dilutions

of antiserum or monoclonal $\mathrm{IgG}(1 \mathrm{mg} / \mathrm{ml})$. Assays were performed as described in Methods. The results given are the average of triplicate determinations. 


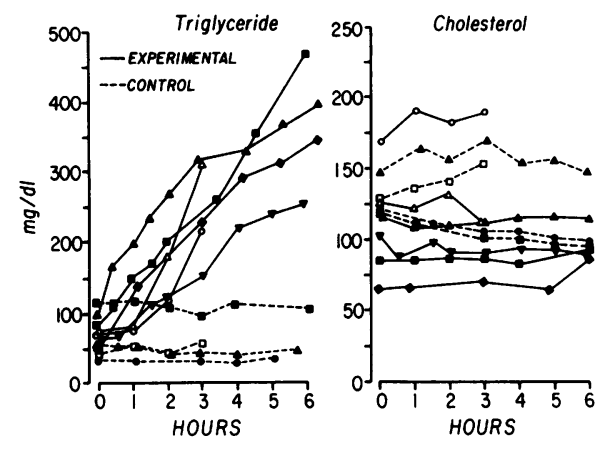

Figure 2. Effect of LPL inhibition on plasma lipid levels. Plasma levels of triglyceride and cholesterol in control monkeys (dashed line) and in monkeys receiving anti-LPL IgG (solid line) are shown.

4, LPL inhibition increased the plasma levels of VLDL particles of different size than those affected by HTGL inhibition. Whereas HTGL inhibition resulted predominantly in an increase in smaller VLDL and IDL, LPL inhibition produced an increase in the mass of larger VLDL particles.

Total plasma cholesterol did not increase during LPL inhibition (Fig. 2). In fact, both LDL and HDL total masses decreased during these studies (Table III) and these changes were associated with decreases in the ratio of cholesterol to TG concentrations in those lipoproteins (Table II). Analytic ultracentrifugation showed a decrease in both $\mathrm{HDL}_{2}$ and $\mathrm{HDL}_{3}$ masses in two animals, whereas in one animal $(C)$, only $\mathrm{HDL}_{3}$ decreased. Total LDL mass decreased. This decrease in LDL was not consistently associated with a decline in the mass of any single subgroup of LDL.

$V L D L$ apoB metabolism. The kinetic curves obtained by injection of radiolabeled human VLDL into control and LPLinhibited monkeys are shown in Fig. 5. The data are presented as the percent of initial VLDL apoB radioactivity remaining in plasma over time. Although the catabolism of VLDL apoB

Table II. Lipoprotein Triglyceride and Cholesterol before and after LPL Inhibition

\begin{tabular}{lllllllll}
\hline & \multicolumn{3}{l}{ Triglyceride } & & & \multicolumn{3}{l}{ Cholesterol } \\
\cline { 2 - 4 } \cline { 6 - 8 } Monkey & 0 & $3 \mathrm{~h}$ & $6 \mathrm{~h}$ & & 0 & $3 \mathrm{~h}$ & $6 \mathrm{~h}$ \\
\hline & $m g / d l$ & $m g / d l$ & $m g / d l$ & & $m g / d l$ & $m g / d l$ & $m g / d l$
\end{tabular}

VLDL

$\begin{array}{rrrrrrl}1 & 9 & 81 & \text { ND } & 3 & 15 & \text { ND } \\ 11 & 37 & 82 & 206 & 6 & 9 & 22 \\ 12 & 44 & 146 & 200 & 6 & 19 & 26 \\ \text { LDL } & & & & & & \\ 1 & 36 & 45 & \text { ND } & 29 & 11 & \text { ND } \\ 11 & 16 & 15 & 19 & 58 & 53 & 42 \\ 12 & 26 & 25 & 22 & 61 & 50 & 33 \\ \text { HDL } & & & & & & \\ 1 & 33 & 36 & \text { ND } & 41 & 21 & \text { ND } \\ 6 & 30 & 40 & \text { ND } & 37 & 32 & \text { ND } \\ 7 & 12 & 15 & 12 & 50 & 21 & 28 \\ 8 & 19 & 26 & 50 & 35 & 10 & 10 \\ 11 & 17 & 27 & 32 & 63 & 49 & 44 \\ 12 & 19 & 19 & 25 & 49 & 23 & 27\end{array}$
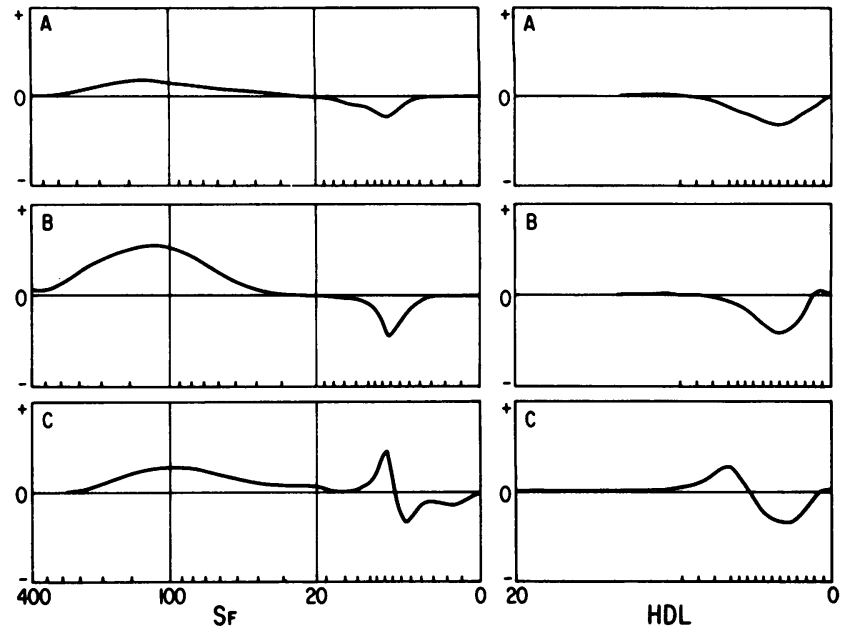

Figure 3. Difference plots of analytical ultracentrifugation illustrating changes in plasma lipoprotein profiles after LPL inhibition in three monkeys. The baseline analytical ultracentrifugal analysis is subtracted from the analysis of the sample obtained $3 \mathrm{~h}$ after beginning infusion of anti-LPL IgG. Deflections above the zero line indicate an increase in mass. Deflections below the line illustrate a loss of mass. The studies shown were performed using monkeys No. 1, 4, and 6.

continued, it appeared to be slower in the LPL-inhibited monkeys. Although the initial component of the die-away curves for VLDL apoB were similar in the control and experimental monkeys, the inflections of the curves occurred earlier in the LPL-inhibited animals. These changes suggested that there was some heterogeneity in the effect of LPL inhibition on VLDL catabolism. VLDL apoB conversion to LDL apoB was completely blocked by LPL inhibition. Therefore, whereas catabolism of some VLDL continued during LPL inhibition, the pathway for conversion of VLDL to LDL appeared to require LPL.

The isolation and injection of tracer amounts of subfractions of human VLDL allowed us to ascertain whether the effect of LPL inhibition was confined to effects on only the more buoyant subclass of VLDL $\left(S_{\mathrm{f}} 400-100\right)$, or was demonstrable in denser VLDL $\left(S_{\mathrm{f}} 60-20\right)$ as well. These studies, shown in Fig. 6, are plotted as percent of initial total VLDL apoB radioactivity remaining in plasma to best illustrate the total amount of VLDL apoB in each subclass that was catabolized and converted to LDL apoB. In the control studies, whereas some apoB was converted to LDL from both subclasses of VLDL, a much greater percent of the $S_{f} 60-20 \mathrm{VLDL}$ was converted to LDL. More buoyant VLDL was, therefore, removed from the circulation without conversion to denser lipoproteins. Although LPL inhibition resulted in a decrease in the removal of VLDL apoB in both $S_{\mathrm{f}} 400-100$ and $S_{\mathrm{f}}$ 60-20 (Fig. $6 \mathrm{~A}$ ) from the VLDL density range, this effect was much more marked for the buoyant $S_{f} 400-100$ particles than for the denser $S_{\mathrm{f}}$ 60-20 VLDL. At the conclusion of the 6-h study, an average of $65 \%$ of the $S_{f} 400-100$ VLDL apoB and $27 \%$ of the $S_{\mathrm{f}}$ 60-20, dense, VLDL apoB remained in VLDL. LPL inhibition also resulted in a marked decrease in conversion of VLDL apoB into IDL apoB (Fig. $6 B$ ) and LDL apoB (Fig. $6 C$ ). Thus, the steps in the VLDL catabolic pathway that were most affected by LPL inhibition were the catabolism of large VLDL and the conversion of smaller VLDL into IDL. 
Table III. Lipoprotein Mass Profiles by Analytical Ultracentrifugation before and after Anti-LPL IgG Infusion

\begin{tabular}{|c|c|c|c|c|c|c|c|c|c|c|c|c|}
\hline \multirow[b]{2}{*}{ Monkey } & \multicolumn{12}{|l|}{$S_{s}$} \\
\hline & 0 & $3 \mathrm{~h}$ & 0 & $3 \mathrm{~h}$ & 0 & $3 \mathrm{~h}$ & 0 & $3 \mathrm{~h}$ & 0 & $3 \mathrm{~h}$ & 0 & $3 \mathrm{~h}$ \\
\hline 1 & 0 & 57 & 2 & 39 & 9 & 12 & 91 & 55 & 179 & 131 & 153 & 108 \\
\hline 4 & 0 & 196 & 2 & 113 & 6 & 4 & 180 & 128 & 229 & 188 & 164 & 109 \\
\hline 6 & 0 & 65 & 0 & 90 & 0 & 2 & 226 & 204 & 309 & 331 & 264 & 212 \\
\hline
\end{tabular}

Metabolism of endogenously labeled apoB. The catabolism of apoB labeled endogenously by injection of $\left[{ }^{3} \mathrm{H}\right]$ leucine was also altered by LPL inhibition. In Fig. 6, a representative study performed in a control animal is shown. In six control animals, $\left[{ }^{3} \mathrm{H}\right]$ leucine radioactivity in plasma VLDL apoB peaked at 60-90 min after injection of the tracer into the monkey. The label was then rapidly removed from VLDL and appeared in LDL (32). The effects of LPL inhibition on endogenously labeled apoB are also shown in Fig. 7. As in control animals, the label appeared in VLDL apoB between 60 and $90 \mathrm{~min}$, but the amount of label that accumulated in VLDL was much greater in the LPL-inhibited animals. Removal of $\left[{ }^{3} \mathrm{H}\right]$ apoB from VLDL was blocked in the LPL-inhibited monkeys, and much of the label remained in VLDL for the duration of the 6-h study. The amount of label that appeared in LDL apoB was $<10 \%$ of the amount appearing in LDL in control studies. LPL inhibition, therefore, almost completely interrupted the production of newly synthesized LDL apoB.

\section{Discussion}

Studies in humans $(13,14)$ and animals $(15)$ have demonstrated that two metabolic processes play critical roles in the catabolism of VLDL TG. Hydrolysis of TG-rich, nascent VLDL generates a series of smaller, more dense VLDL. Some of these VLDL remnant particles are removed directly from the circulation via interaction with the B-E receptor $(33,34)$ and possibly another lipoprotein receptor in the liver (35).
Approximately $50 \%$ of the VLDL particles, however, are converted to $\operatorname{LDL}(14,15)$. The role of enzymatic hydrolysis of TG in the regulation of the catabolism of more buoyant and more dense VLDL subclasses in vivo, and of the conversion of VLDL to LDL has not been clearly elucidated. Moreover, whereas each VLDL may have the option to be metabolized to LDL or to be removed from plasma as VLDL, an alternative hypothesis that remains to be tested is that there are discrete subpopulations of VLDL which undergo catabolism by only one of these pathways (referred to as metabolic channeling) and that only some VLDL interact with LPL.

The present studies of acute LPL inhibition in the monkey clarify the role of that enzyme in the catabolism of subclasses of VLDL and in the production of LDL. LPL inhibition resulted in a marked increase in the more buoyant $\left(S_{\mathrm{f}} 400-100\right)$ VLDL. The kinetic studies after the injection of radiolabeled VLDL confirmed that the increase in more buoyant VLDL was due (at least in part) to a defect in catabolism of this subclass of VLDL particles. These results demonstrated that larger, buoyant VLDL, in a manner analogous to chylomicrons, require LPL to initiate the catabolic pathway resulting in their removal from the circulation. This increase in mass may also have resulted from an increased synthesis of larger VLDL secondary to the return to the liver of more TG-rich remnants, which were incompletely hydrolyzed in the periphery. In contrast, inhibition of LPL activity had only a modest effect on the rate of catabolism of VLDL $\mathrm{S}_{\mathrm{f}} 60-20$. These data support a role for a non-LPL-mediated pathway in catabolism of smaller VLDL. Our previous studies and the recent studies
A
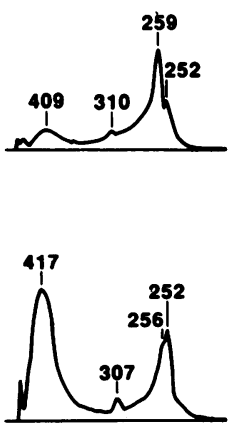

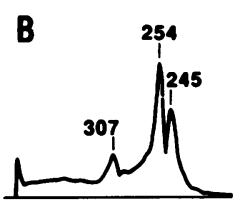

C
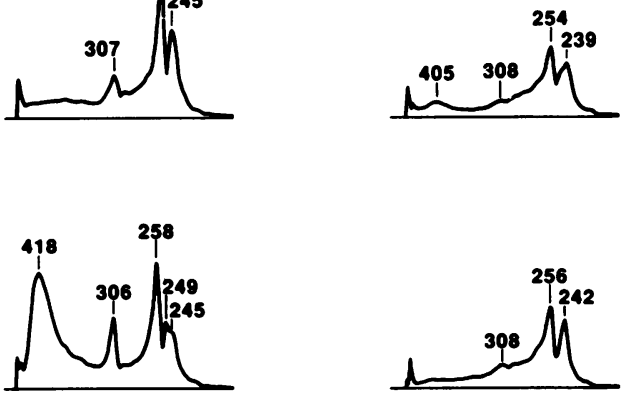

D
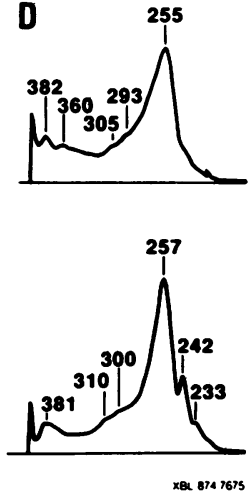

Figure 4. Nondenaturing $2-16 \%$ polyacrylamide gradient gel electrophoresis of the $d<1.063 \mathrm{~g} / \mathrm{ml}$ lipoprotein fraction from four monkeys before (top row) and $3 \mathrm{~h}$ after (bottom row) infusion of $\mathrm{IgG}$. $(A$ and $B$ ) Monkeys injected with anti-lipoprotein lipase IgG. $(C)$ Monkey injected with control IgG. (D) Monkey injected with anti-hepatic lipase IgG. Electrophoresis was carried out, and gels were stained with oil red $\mathrm{O}$ and scanned as described previously $(25,26)$. 


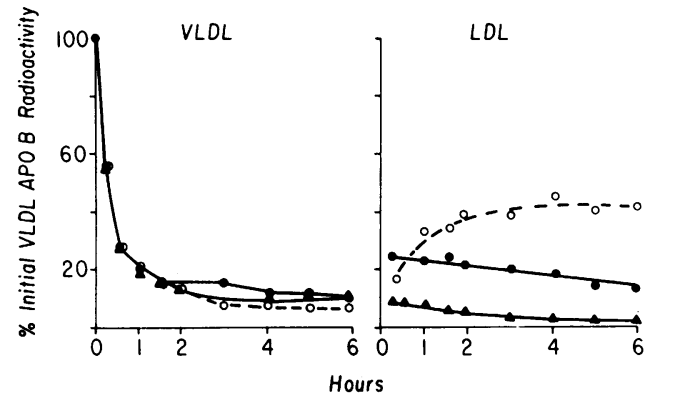

Figure 5. Effect of LPL inhibition on VLDL apoB metabolism. The apoB radioactivity decay curves in VLDL and LDL, plotted as percent of initial apoB radioactivity in VLDL, are graphed vs. time. Control animals are shown using dashed lines and LPL inhibited monkeys are shown using solid lines. The studies were performed using monkeys No. 7, 8, and 9 .
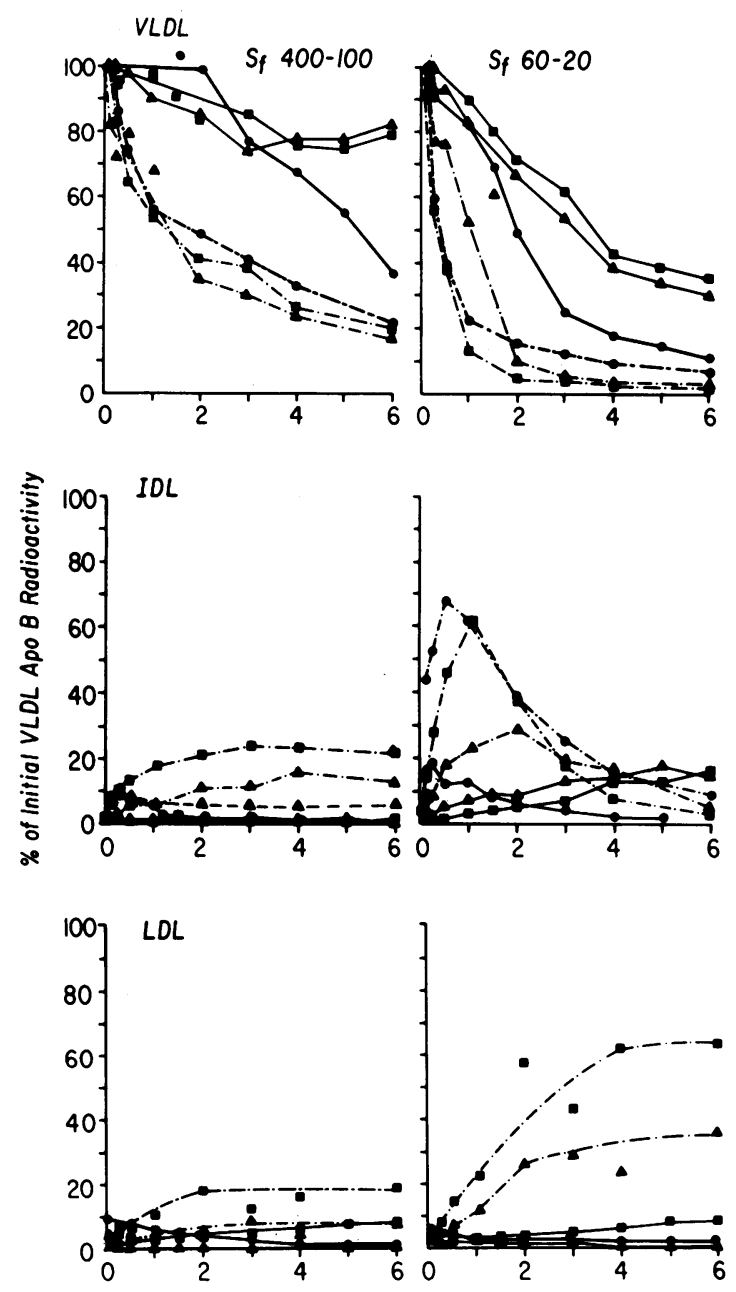

Figure 6. The metabolism of buoyant VLDL, $\mathrm{S}_{\mathrm{f}} 400-100$, and dense VLDL, $S_{\mathrm{f}} 60-20$, in control and LPL inhibited monkeys. Data are presented as percent of initial VLDL apoB radioactivity per milliliter of plasma. Control studies are shown in dotted lines and studies in which animals received anti-LPL IgG are shown in solid lines. Each animal is shown using the same symbol for its control and experimental studies. Monkeys No. 10, 11, and 12 were used for these studies.

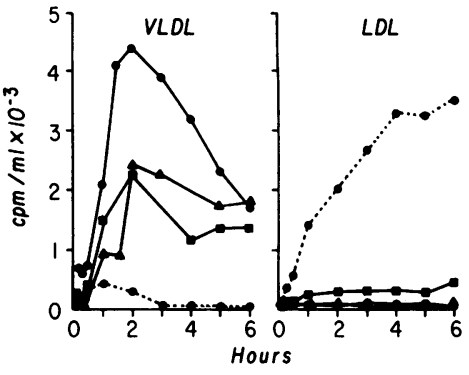

Figure 7. Metabolism of endogenously labeled apoB in control and LPL inhibited monkeys. $\left[{ }^{3} \mathrm{H}\right]$ Leucine was intravenously injected into the monkeys and the radioactivity in VLDL and LDL apoB per microliter of plasma are graphed vs. time. Studies in LPL-inhibited monkeys (solid lines)

were performed in animals No. 3, 5, and 11. A representative study performed in a control animal is shown (dotted line) for comparison.

of Manzato et al. (36) and Berry et al. (37) support a role for HTGL in the catabolism of smaller VLDL and IDL. Our current results imply that significant quantities of TG in dense VLDL are hydrolyzed by HTGL. A more quantitative assessment of the degradation rates of TG-rich lipoproteins would require studies performed during chronic LPL inhibition in which a new steady-state level of plasma lipoproteins had been achieved.

As has been previously demonstrated by Packard et al. (38) and Stalenhoef et al. (39), a greater proportion of the apoB label in buoyant VLDL, as compared with denser VLDL, was removed from the circulation without conversion to LDL. If hypotheses proposed concerning metabolic channeling are correct $(38,39)$, buoyant VLDL would be a poor substrate for conversion to LDL. Alternatively, the limited conversion of these particles to LDL may be the result of competition along many steps of the lipolytic cascade for pathways either resulting in VLDL removal or conversion to LDL. As noted above, in comparison to the conversion of VLDL $S_{\mathrm{f}} 400-100$, a larger percent of the more dense VLDL, $S_{\mathrm{f}} 60-20$, apoB was converted to LDL in the normal monkey. Conversion of dense VLDL to LDL was, however, completely inhibited when LPL activity was blocked in the monkey. These data demonstrated that LPL was required not only for the initiation of catabolism of large (perhaps nascent) VLDL, but that smaller, dense VLDL also required an interaction with this enzyme for their conversion to LDL. LPL inhibition resulted in removal of a much greater percentage of the $S_{f} 60-20$ VLDL apoB from the circulation without conversion to LDL, demonstrating that some dense VLDL were able to be catabolized by either of two pathways. Moreover, when one pathway was less efficient or completely blocked, as in these studies, more particles were catabolized by the alternative route. Conversion of buoyant $\left(\mathbf{S}_{\mathbf{f}}\right.$ 400-100) VLDL to LDL was also reduced during LPL inhibition and there was continued removal of some buoyant LDL from the circulation. Although no direct data is available on the tissue sites of removal of these lipoproteins, the accumulation of lipid in the cells of the reticuloendothelial system in LPL-deficient humans suggests that some of these particles may be found in similar cells in the experimental monkeys.

Conversion of VLDL to IDL was retarded by LPL inhibition, and production of LDL was inhibited completely. A number of experimental studies imply that LPL alone is insufficient to produce LDL from its TG-rich precursor lipoproteins. In studies performed in vitro, Deckelbaum et al. produced particles which were slightly larger than normal LDL by hydrolysis of VLDL TG using bovine LPL (11). We have shown that in human PHP, in which intravascular hydrolysis 
of lipoproteins was accelerated by released lipase enzymes, LPL was associated with lipoproteins which were slightly larger than LDL (40). Rubinstein et al. studied the shift of apoE in plasma obtained before and after heparin in subjects with genetic deficiencies of either LPL or HTGL (41). LPL-deficient subjects did not produce the apoE-rich particles that are slightly larger than LDL, suggesting that these lipoproteins are the metabolic product of LPL-mediated hydrolysis of VLDL. Production of these E-rich particles, which may be the usual precursors of LDL, was blocked in our current studies resulting in a block of LDL production. Additional steps (not addressed by our current studies) are necessary for final conversion of these particles to circulating LDL. These steps may include remodeling of the lipoprotein by HTGL, transfer proteins, or by interaction with cell-surface receptors.

These studies of acute LPL inhibition can be compared with the studies of VLDL apoB metabolism in subjects with type I hyperlipoproteinemia reported by Nicoll and Lewis (6). Both our studies and those of Nicoll and Lewis show continued catabolism of circulating VLDL despite the absence of LPL activity. In the human studies (6), the authors noted that conversion of VLDL apoB to LDL continued in one of their subjects who was deficient in apoCII, the activator of LPL. Because, in our studies, the most dramatic finding was the complete inhibition of VLDL apoB conversion to LDL, it is possible that the studies in the apoCII deficient patient demonstrated effects due to the action of a small amount of LPL activity or the presence of normal LPL protein which allowed this conversion to continue, perhaps via nonenzymatic actions of LPL. In this regard, LPL has been shown in some situations to functionally act as a transfer protein for cholesteryl esters (42). As noted above, this action may be needed to produce LDL.

The studies using $\left[{ }^{3} \mathrm{H}\right]$ leucine to endogenously label apoB confirmed that newly synthesized VLDL required LPL to initiate their catabolism. Thus, the disappearance of the ${ }^{3} \mathrm{H}$ label from apoB in VLDL was markedly retarded during LPL inhibition. Furthermore, because virtually no labeled apoB appeared in LDL during LPL inhibition, direct synthesis of LDL by the monkey liver appeared to be insignificant. Although we had previously reported that kinetic modeling of combined VLDL and LDL turnover studies in the monkey showed that $30-50 \%$ of the LDL was not derived from circulating VLDL apoB (15), the present studies demonstrated that, in the LPLinhibited chow-fed monkey, there was minimal independent secretion of LDL by the liver. This result is consistent with the studies of Johnson et al. (43), who showed that the livers of high-cholesterol, high-fat fed monkeys secreted relatively few lipoproteins the size of LDL. Similarly, these data obtained during LPL inhibition complement our recent kinetic studies comparing the metabolism of apoB endogenously labeled with $\left[{ }^{3} \mathrm{H}\right]$ leucine and VLDL exogenously labeled with radioiodine (32). Kinetic modeling of those studies demonstrated the presence of a pool of VLDL which was rapidly converted to LDL and which appeared to bypass the pool of circulating VLDL. Whereas the VLDL particles which are in this pool may differ in composition from those in circulating VLDL, it is equally plausible that by random chance a given VLDL may interact in such a manner with LPL (either on the endothelial surface or in the plasma) that core TG is rapidly and almost completely hydrolyzed in a single step, producing a particle which is no longer isolated in the VLDL density range. If such a pool of rapidly hydrolyzed VLDL occurs in humans it would account for the apparent direct production of LDL that has been observed in kinetic tracer studies in human lipoprotein disorders (13) and during physiological manipulations (14). It should be noted that the rapid elevation of plasma TG which occurred in our studies during LPL inhibition could have resulted in a net increase in TG uptake by the liver. In turn, an increase in the TG content of newly secreted lipoproteins would have been expected resulting in the secretion of larger VLDL which were less likely to be converted to LDL. Thus, these studies of endogenously produced lipoproteins may not totally reflect the synthesis of lipoproteins by livers of control animals.

The levels of circulating HDL cholesterol in humans are regulated, at least in part, by the actions of LPL, because lipolysis of TG-rich lipoproteins produces surface components that are transferred to HDL (44). HDL in the monkey normally has a fractional catabolic rate of $0.35-0.38$ pools per $\mathrm{d}(45,46)$. The rapid decrease of HDL cholesterol to $50 \%$ of its initial level in LPL-inhibited animals, therefore, suggested that HDL cholesterol removal was occurring by mechanisms other than metabolism of intact HDL particles. As HDL cholesterol decreased, HDL TG increased, perhaps because HDL cholesterol was exchanged for TG found in the increased pool of VLDL. A reduction of the mass of HDL was also demonstrated in analytical ultracentrifugation profiles. Inhibition of LPL, therefore, resulted in a concomitant elevation of TG-rich lipoproteins and decrease in HDL cholesterol, producing a lipoprotein profile similar to that found in humans with type IV hyperlipoproteinemia who often have low HDL cholesterol and hypertriglyceridemia.

\section{Acknowledgments}

The authors thank Benjamin Leeman, Theresa Vanni, and Steven Wong for their technical assistance.

This work was supported by grants HL 21006 (SCOR) and HL 36000 from the National Heart, Lung, and Blood Institute and a Grant-In-Aid from the American Heart Association. Dr. Goldberg was the recipient of a Clinician Scientist Award from the American Heart Association and its local affiliate the New York Heart Association.

\section{References}

1. Fredrickson, D. S., J. L. Goldstein, and M. S. Brown. 1978. The Familial Hyperlipoproteinemias. The Metabolic Basis of Inherited Disease. J. B. Stanbury, J. B. Wyngaarden, and D. S. Fredrickson, editors. McGraw-Hill Book Co., New York. 608-617.

2. Havel, R. J., and R. J. Gordon, Jr. 1960. Idiopathic hyperlipidemia: metabolic studies in an affected family. J. Clin. Invest. 39:17771790.

3. Kompiang, I. D., A. Bensadoun, and M.-W. W. Yang. 1976. Effect of an anti-lipoprotein lipase serum on plasma triglyceride removal. J. Lipid Res. 17:498-505.

4. Ford, S., Jr., W. K. Schubert, C. J. Glueck, and R. C. Bozian. 1971. Familial hyperchylomicronemia: enzymatic and physiologic studies. Am. J. Med. 50:536-541.

5. Quarfordt, S. M., A. Frank, D. M. Shames, M. Berman, and D. Steinberg. 1970. Very low density lipoprotein triglyceride transport in type IV hyperlipoproteinemia and the effects of carbohydrate rich diets. J. Clin. Invest. 49:2281-2297.

6. Nicoll, A. B., and B. Lewis. 1980. Evaluation of the roles of lipoprotein lipase and hepatic lipase in lipoprotein metabolism in vivo and in vitro studies. Eur. J. Clin. Invest. 10:489-495.

7. Brunzell, J. D., W. R. Hazzard, D. Porte, Jr., and E. L. Bierman. 
1973. Evidence for a common saturable triglyceride removal mechanism for chylomicrons and very low density lipoprotein in man. $J$. Clin. Invest. 52:1578-1585.

8. Goldberg, I. J., N. A. Le, J. R. Paterniti, H. N. Ginsberg, F. T. Lindgren, and W. V. Brown. 1982. Lipoprotein metabolism during acute inhibition of hepatic triglyceride lipase in the cynomolgus monkey. J. Clin. Invest. 70:1184-1192.

9. Grosser, J., O. Schrecker, and H. Greten. 1981. Function of hepatic triglyceride lipase in lipoprotein metabolism. J. Lipid Res. 22:437-442.

10. Rao, S. N., C. Cortese, N. E. Miller, Y. Levy, and B. Lewis. 1982. Effects of heparin infusion on plasma lipoproteins in subjects with lipoprotein lipase deficiency. FEBS (Fed. Eur. Biochem. Soc.) Lett. 150:255-259.

11. Deckelbaum, R. J., S. Eisenberg, M. Fainaru, L. Barelholz, and T. Olivecrona. 1979. In vitro production of human plasma low density lipoprotein-like particles. J. Biol. Chem. 254:6079-6087.

12. Musliner, T. A., P. N. Herbert, and M. J. Kingston. 1979. Lipoprotein substrates of lipoprotein lipase and hepatic triacylglycerol lipase from human postheparin plasma. Biochim. Biophys. Acta. 575:277-288.

13. Janus, E. D., A. Nicoll, R. Wooton, P. R. Turner, P. J. Magill, and B. Lewis. 1980. Quantitative studies of very low density lipoprotein in normal controls and primary hyperlipoproteinemic states and the role of direct secretion of low density lipoprotein in heterozygous familial hypercholesterolaemia. Eur. J. Clin. Invest. 10:149-159.

14. Ginsberg, H. N., N. A. Le, and J. Corey-Gibson. 1985. Regulation of the production and catabolism of low density lipoproteins in hypertriglyceridemic subjects. Effects of weight loss. J. Clin. Invest. 75:614-623.

15. Goldberg, I. J., N. A. Le, H. N. Ginsberg, J. R. Paterniti, and W. V. Brown. 1983. Metabolism of apolipoprotein B in the cynomolgus monkey. Am. J. Physiol. 244:E196-E201.

16. Goldberg, I. J., J. R. Paterniti, and W. V. Brown. 1983. Measurement of post-heparin lipase enzymes in the cynomolgus monkey. Biochim. Biophys. Acta. 752:172-177.

17. Goldberg, I. J., R. S. Rosenfeld, I. Paul, and B. Leeman. 1986. Generation of plasma-free cholesterol from circulating lipoprotein associated cholesteryl ester. Am. J. Phys. 250:E265-E268.

18. Thomas, M. S., and L. L. Rudel. 1983. [ $\left.{ }^{3} \mathrm{H}\right]$ Cholesteryl ester labeling and transfer among human and nonhuman primate plasma lipoproteins. Anal. Biochem. 130:215-222.

19. Finley, P. R., R. B. Schifman, R. J. Williams, and D. A. Lichti. 1978. Cholesterol in high density lipoprotein: use of $\mathrm{Mg}^{2+} /$ dextran sulfate in its enzymic measurement. Clin. Chem. 24:931-933.

20. Goldberg, I. J., W. S. Blaner, and D. S. Goodman. 1986. Immunological and enzymatic comparisons between human and bovine lipoprotein lipase. Arch. Biochem. Biophys. 244:580-584.

21. Goldberg, I. J., J. R. Paterniti, D. S. France, G. Martinelli, and J. A. Cornicelli. 1986. Production and use of a monoclonal antibody to human lipoprotein lipase. Biochim. Biophys. Acta. 878:168-176.

22. Hybridoma Techniques. Cold Spring Harbor Laboratory, Cold Spring Harbor, NY.

23. Nilsson-Ehle, P., and M. D. Schotz. 1976. A stable, radioactive substrate emulsion for assay of lipoprotein lipase. J. Lipid Res. 17:536-541.

24. Lindgren, F. T., L. D. Jensen, and F. T. Hatch. 1972. The isolation and quantitative analysis of serum lipoproteins. In Blood Lipids and Lipoproteins. G. J. Nelson, editor. John Wiley \& Sons, Inc., New York. 181-274.

25. Krauss, R. M., and D. J. Burke. 1982. Identification of multiple subclasses of plasma low density lipoproteins in normal humans. $J$. Lipid Res. 23:97-104.

26. Musliner, T. A., C. Giotas, and R. M. Krauss. 1986. Presence of multiple subpopulations of lipoproteins of intermediate density in normal subjects. Arteriosclerosis. 6:79-87.

27. MacFarlane, A. S. 1958. Efficient trace labeling of proteins with iodine. Nature (Lond.). 182:53.
28. Bilheimer, D. W., S. Eisenberg, and R. I. Levy. 1972. The metabolism of very low density lipoprotein proteins. 1. Preliminary in vitro and in vivo observations. Biochim. Biophys. Acta. 260:212-221.

29. Havel, R. J., H. A. Eder, and J. H. Bragdon. 1955. The distribution and chemical composition of ultracentrifugally separated lipoproteins in human serum. J. Clin. Invest. 34:1345-1353.

30. Le, N. A., J. S. Melish, B. C. Roach, H. N. Ginberg, and W. V. Brown. 1978. Direct measurement of apo-protein B specific activity in ${ }^{125}$ I-labeled lipoproteins. J. Lipid Res. 19:578-584.

31. Lowry, O. H., N. J. Rosebrough, A. L. Farr, and R. J. Randall. 1951. Protein measurement with the Folin phenol reagent. J. Biol. Chem. 193:267-275.

32. Le, N. A., I. J. Goldberg, H. N. Ginsberg, and W. V. Brown. 1983. Direct production of low density lipoprotein in the monkey. Comparison of endogenous versus exogenous tracers. Arteriosclerosis. 3:503. (Abstr.)

33. Krul, E. S., M. J. Tikhanen, T. G. Cole, J. M. Davies, and G. Schonfeld. 1985. Roles of apolipoproteins B and E in the cellular binding of very low density lipoproteins. J. Clin. Invest. 75:361-369.

34. Gianturco, S. H., A. M. Gotto, Jr., S.-L. H. Wang, J. B. Karlin, A. H. Y. Lin, S. C. Prasad, and W. A. Bradley. 1983. Apolipoprotein E mediates uptake of $S_{f} 100-400$ hypertriglyceridemic very low density lipoproteins by the low density lipoprotein receptor pathway in normal human fibroblasts. J. Biol. Chem. 258:4526-4533.

35. Hui, D. Y., T. L. Innerarity, and R. W. Mahley. 1981. Lipoprotein binding to canine hepatic membranes. J. Biol. Chem. 256:5646-5655.

36. Manzato, E., S. Zambon, R. Marin, G. Baggio, and G. Crepaldi. 1986. Modifications of plasma lipoproteins after lipase activation in patients with chylomicronemia. J. Lipid Res. 27:1248-1258.

37. Berry, E. M., R. Aldinia, H. Bar-On, and S. Eisenberg. 1981. Role of the liver in degradation of very low density lipoproteins. A study of lipolysis by heparin releasable liver lipase and uptake during isolated rat liver perfusion. Eur. J. Clin. Invest. 11:151-159.

38. Packard, C. J., A. Munro, A. R. Lorimer, A. M. Gotto, and J. Shepherd. 1984. Metabolism of apolipoprotein B in large triglyceriderich very low density lipoproteins of normal and hypertriglyceridemic subjects. J. Clin. Invest. 74:2178-2192.

39. Stalenhoef, A. F. H., M. J. Malloy, J. P. Kane, and R. J. Havel. 1984. Metabolism of apolipoproteins B-48 and B-100 of triglyceriderich lipoproteins in normal and lipoprotein lipase-deficient humans. Proc. Natl. Acad. Sci. USA. 81:1839-1843.

40. Goldberg, I. J., J. J. Kandel, C. B. Blum, and H. N. Ginsberg. 1986. Association of plasma lipoproteins with postheparin plasma lipase activities. J. Clin. Invest. 78:1523-1528.

41. Rubinstein, A., J. C. Gibson, J. R. Paterniti, Jr., G. Kaksis, A. Little, H. N. Ginsberg, and W. V. Brown. 1985. Effect of heparin-induced lipolysis on the distribution of apolipoprotein $\mathrm{E}$ among lipoprotein subclasses. J. Clin. Invest. 75:710-721.

42. Friedman, G., T. Chajek-Shaul, O. Stein, T. Olivecrona, and Y. Stein. 1981. The role of lipoprotein lipase in the assimilation of cholesteryl linoleyl ether by culture cells incubated with labeled chylomicrons. Biochim. Biophys. Acta. 666:156-164.

43. Johnson, F. L., R. W. Clara, and L. L. Rudel. 1983. Studies on the production of low density lipoproteins by perfused livers from nonhuman primates; effects of dietary cholesterol. J. Clin. Invest. 72:221-236.

44. Eisenberg, S., and T. Olivecrona. 1979. Very low density lipoprotein. Fate of phospholipids, cholesterol and apolipoprotein $\mathrm{C}$ during lipolysis in vitro. J. Lipid Res. 20:614-623.

45. Kushwaha, R. S., D. M. Foster, and W. R. Hazzard. 1982. Effect of diet-induced hypercholesterolemia on high density lipoprotein metabolism in pigtail monkeys (maccaca Memestrina). Metabolism. 31:43-51.

46. Parks, J. S., and L. L. Rudel. 1982. Different kinetic fates of apolipoproteins A-I and A-II from lymph chylomicra of nonhuman primates. Effect of saturated versus polyunsaturated dietary fat. $J$. Lipid Res. 23:410-412. 